\title{
Collisionless dynamics of zonal flows in stellarator geometry
}

\author{
Alexey Mishchenko*, Per Helander and Axel Könies \\ Max-Planck-Institut für Plasmaphysik, \\ EURATOM-Association, D-17491 Greifswald, Germany
}

(Dated: June 30, 2008)

\begin{abstract}
The collisionless time evolution of zonal flows in stellarator systems is investigated. An analytical solution of the kinetic and quasineutrality equations describing the residual zonal flow is derived for arbitrary three-dimensional systems without approximations in the magnetic geometry. The theory allows for an arbitrary number of particle species. It has been found that in stellarators the residual zonal flows are not in general steady but oscillate with a certain frequency. This frequency is determined by the speed of the bounce-averaged radial drifts of the particles trapped in the magnetic field and vanishes in tokamaks, where such net drifts are absent. A reduction of the bounce-averaged radial drifts in configurations optimized with respect to neoclassical transport results in a smaller zonal-flow frequency.
\end{abstract}

\footnotetext{
* alexey.mishchenko@ipp.mpg.de
} 


\section{INTRODUCTION}

Stellarator systems are known to have larger particle losses than tokamaks, because of the lack of symmetry resulting in bounce-averaged radial drifts of helically-trapped particles. The reduction of these particle losses is a necessary condition for the realization of fusion in non-axisymmetric devices. Since the early 1980s, a range of approaches to the optimization of the stellarator geometry has been developed $[1,2]$. One of the goals of this optimization has been a reduction of the neoclassical transport. As a result, interest has also arisen to reduce the turbulent transport. As for tokamaks, it is believed that an important mechanism regulating the transport caused by the microinstabilities are the so-called zonal flows [3], which involve $\mathbf{E} \times \mathbf{B}$ flows due to a radially varying electrostatic potential $\phi(r, t)$ driven by the nonlinearities in the kinetic equation. It is known that zonal flows are partially shielded due to the finite banana-orbit width of the particles (resulting in so-called neoclassical polarization [4]). In this respect, it is important to know how large the residual flow is because, to some extent, this flow indicates how effectively the turbulence can be suppressed. It is also of interest to study how the magnetic geometry affects the level of the residual zonal flow. Being well developed in tokamaks [4-7], the theory of zonal flow shielding due to neoclassical plasma polarization is under development in stellarators [8-12].

A strong interest in the dependence of the zonal flow shielding on the magnetic geometry has been triggered by recent experimental results from the Large Helical Device [13] (LHD) where it has been observed that not only neoclassical but also anomalous transport is reduced by an inward shift of the magnetic axis. This decreases the radial drift of helically-trapped particles but also increases the unfavorable magnetic curvature to destabilize pressure-gradient-driven instabilities such as the ITG mode (see Ref. [8] and the papers referenced therein). It has been argued that the drift optimization is closely correlated with the optimization of the residual zonal flow level. Thus, the larger linear growth rates of the ITG modes in the LHD configuration with the inward shift of the magnetic axis can be compensated by more effective turbulence suppression through a larger zonal flow. To support this argumentation, the kinetic theory of the linear evolution of zonal flows in multiple-helicity systems has been developed in Refs. [8, 9] (employing some approximations with respect to the magnetic geometry). This theory has shown that bounce-averaged radial drifts play an important role in the collisionless long-time evolution of zonal flows. 
Also Ref. [12], where the action-angle formalism is used to solve the kinetic equation, has demonstrated a close link between the particle radial drifts and the value of the residual zonal flow.

In this paper, we develop a kinetic theory of the linear evolution of zonal flows in arbitrary three-dimensional geometry (assuming that flux surfaces exist). We consider the long-time evolution of the zonal flow (i.e. we assume the bounce time to be much smaller than the characteristic time of the zonal flow). We solve the kinetic equation in guiding-center coordinates similar to Refs. [8, 9], however, we do not rely on approximations in the magnetic field geometry. As we shall see, the residual zonal flow resulting from the analytical solution of the kinetic equation can be expressed in terms of some flux-surface and orbit averages. We compute these geometry-related quantities numerically.

The structure of the paper is as follows. In Sec. II, basic equations for describing zonal flows in stellarator geometry are given. In Secs. III and IV, we solve these equations analytically. In Sec. V, the numerical approach to the geometry-related quantities is presented. In Sec. VI, we apply our theory and numerical algorithm to the Large Helical Device [13] and the Wendelstein 7-X stellarator [14]. Finally, we discuss the results of our calculations and draw conclusions.

\section{BASIC EQUATIONS}

The basic equations for the Rosenbluth-Hinton theory $[4,5]$ is the coupled system of the linear gyro-kinetic equation and the quasineutrality equation for the self-consistent electrostatic field potential:

$$
\frac{\partial f_{a 1}}{\partial t}+v_{\|} \nabla_{\|} f_{a 1}+\mathbf{v}_{\mathbf{d}} \cdot \nabla f_{a 1}=-\dot{\varepsilon} \frac{\partial f_{a 0}}{\partial \varepsilon}, \quad-\sum_{a} \nabla \cdot\left(\frac{e_{a}^{2} n_{0 a}}{T_{a}} \rho_{a}^{2} \nabla_{\perp} \phi\right)=\sum_{a} e_{a} n_{a},
$$

where $\varepsilon=m v^{2} / 2$ is the kinetic energy, $\rho_{a}=\sqrt{m_{a} T_{a}} /\left(e_{a} B\right)$ is the thermal gyroradius and $n_{0 a}$ is the equilibrium density of the species $a$. The kinetic energy changes due to the electrostatic field perturbation $\dot{\varepsilon}=-e \mathbf{v}_{\mathbf{d}} \cdot \nabla \phi$ with $\mathbf{v}_{\mathbf{d}}=\rho_{\|} \nabla \times \mathbf{v}_{\|}$being the particle drift velocity and $\rho_{\|}=v_{\|} / \omega_{c}$ being the parallel gyroradius. It is assumed that the characteristic scale of the zonal flow is larger than the ion gyroradius. The sums in the quasineutrality equation are taken over the particle species (an arbitrary number of species is allowed).

As we are to consider the long-time evolution of the residual flow (on a time scale much 
slower than the bounce time), the evolution of the electrostatic potential inside a flux surface can be neglected because it occurs on the bounce time scale. Consequently, the perturbed electrostatic potential depends on the flux-surface label $s$ and time $t$ only, $\phi=\phi(s, t)$. Applying this assumption to the kinetic equation (1) gives:

$$
\frac{\partial f_{a 1}}{\partial t}+v_{\|} \nabla_{\|} f_{a 1}+\mathbf{v}_{\mathbf{d}} \cdot \nabla f_{a 1}=-\frac{e_{a} \phi^{\prime}}{T_{a}} f_{a 0}\left(\mathbf{v}_{\mathbf{d}} \cdot \nabla s\right)
$$

The quasineutrality equation can be rewritten using the relation:

$$
\left\langle\nabla \cdot\left(\frac{e_{a}^{2} n_{0 a}}{T_{a}} \rho_{a}^{2} \nabla_{\perp} \phi\right)\right\rangle=\frac{1}{V^{\prime}} \frac{\mathrm{d}}{\mathrm{d} s}\left[V^{\prime}\left\langle\rho_{a}^{2}|\nabla s|^{2}\right\rangle n_{0 a} \frac{e_{a}^{2} \phi^{\prime}}{T_{a}}\right] .
$$

Here, $\phi^{\prime}=\partial \phi / \partial s, V^{\prime}=\mathrm{d} V / \mathrm{d} s, V$ is the magnetic volume inside the flux surface $s$ and $\langle\ldots\rangle$ is the flux-surface average. Substituting Eq. (3) in Eq. (1), results in a quasineutrality equation in the following form:

$$
-\frac{1}{V^{\prime}} \frac{\mathrm{d}}{\mathrm{d} s}\left[V^{\prime} \sum_{a}\left\langle\rho_{a}^{2}|\nabla s|^{2}\right\rangle \frac{n_{0 a} e_{a}^{2}}{T_{a}} \phi^{\prime}\right]=\sum_{a} e_{a}\left\langle n_{a}\right\rangle .
$$

In Boozer coordinates [2, 15], the magnetic field can be written as

$$
\mathbf{B}=F_{T}^{\prime} \nabla s \times \nabla \theta+F_{P}^{\prime} \nabla \varphi \times \nabla s=J \nabla \theta+I \nabla \varphi+\tilde{\beta} \nabla s
$$

with the toroidal flux $F_{T}^{\prime}$, poloidal flux $F_{P}^{\prime}$, toroidal current $J$, poloidal current $I$ and Boozer angles $\theta$ and $\varphi$. The derivative along the magnetic field line and the radial projection of the drift velocity take the form:

$$
v_{\|} \nabla_{\|}=\frac{v_{\|}}{B \sqrt{g}}\left(F_{P}^{\prime} \partial_{\theta}+F_{T}^{\prime} \partial_{\varphi}\right), \quad \mathbf{v}_{\mathbf{d}} \cdot \nabla s=\frac{v_{\|}}{B \sqrt{g}}\left(I \partial_{\theta}-J \partial_{\varphi}\right) \rho_{\|},
$$

where $\sqrt{g}=[(\nabla s \times \nabla \theta) \cdot \nabla \varphi]^{-1}$ is the Jacobian.

We define an orbit-average operation $\bar{A}$ that annihilates the differential operator $v_{\|} \nabla_{\|}$, so that $\overline{v_{\|} \nabla_{\|} f}=0$ for any function $f$. Note that for the trapped particles this operation coincides with the bounce average and is given in Boozer coordinates by the expression:

$$
\left.\bar{A}\right|_{\text {trapped }}=\left(\oint A \frac{B \sqrt{g}}{v_{\|} F_{P}^{\prime}} \mathrm{d} \theta\right) /\left(\oint \frac{B \sqrt{g}}{v_{\|} F_{P}^{\prime}} \mathrm{d} \theta\right),
$$

where the integrals are computed back and forth between the reflecting points. For passing particles, the orbit-average operation can be defined through the flux-surface average:

$$
\left.\bar{A}\right|_{\text {passing }}=\frac{\left\langle A B /\left|v_{\|}\right|\right\rangle}{\left\langle B /\left|v_{\|}\right|\right\rangle}
$$


Extracting an orbit-averaged part out of the radial drift velocity, one can write it as a sum of "averaged" (slow) and "oscillating" (fast) parts:

$$
\mathbf{v}_{\mathbf{d}} \cdot \nabla s=\overline{\mathbf{v}_{\mathbf{d}} \cdot \nabla s}+v_{\|} \nabla_{\|} G .
$$

Here, the quantity $G$ can be found from the following "magnetic differential equation":

$$
v_{\|} \nabla_{\|} G=\widetilde{\omega}_{r}, \quad \widetilde{\omega}_{r}=\omega_{r}-\bar{\omega}_{r}
$$

The notation $\omega_{r}=\mathbf{v}_{\mathbf{d}} \cdot \nabla s$ has been employed. One can show that in the case of trapped particles $\bar{\omega}_{r} \sim \partial J_{\|} / \partial \alpha$ where $J_{\|}$is the second adiabatic invariant and $\alpha=\varphi-q \theta$ is the field-line label. Recall that the derivative $\partial J_{\|} / \partial \alpha$ is related to the radial precession of locally-trapped particles in non-axisymmetric geometries. For passing particles, one finds:

$$
\bar{\omega}_{r} \propto\left\langle\frac{B \mathbf{v}_{\mathbf{d}}}{v_{\|}} \cdot \nabla s\right\rangle=0 .
$$

\section{COLLISIONLESS THEORY OF RESIDUAL ZONAL FLOW}

Integrating the gyrokinetic equation Eq. (2) over velocity space and averaging the result over the flux surface, one obtains the continuity equation:

$$
\frac{\partial\left\langle n_{a}\right\rangle}{\partial t}+\left\langle\int \mathrm{d}^{3} v \mathbf{v}_{\mathrm{d} a} \cdot \nabla f_{a 1}\right\rangle=0
$$

Taking into account that $\nabla \cdot\left(B \mathbf{v}_{\mathbf{d}} / v_{\|}\right)=0$ (which follows from the relation $\left.\mathbf{v}_{\mathbf{d}}=\rho_{\|} \nabla \times \mathbf{v}_{\|}\right)$, one can rewrite the second term in Eq. (12) in the following form:

$$
\left\langle\int \mathrm{d}^{3} v \mathbf{v}_{\mathbf{d}} \cdot \nabla f_{a 1}\right\rangle=\frac{1}{V^{\prime}} \frac{\mathrm{d}}{\mathrm{d} s} V^{\prime} \pi \sum_{\sigma} \int\left\langle\Theta\left(\xi^{2}\right) \frac{B}{v_{\|}} f_{a 1}\left(\mathbf{v}_{\mathbf{d}} \cdot \nabla s\right)\right\rangle \sigma v^{3} \mathrm{~d} v \mathrm{~d} \lambda .
$$

Here, $\xi^{2}=1-\lambda B, \Theta\left(\xi^{2}\right)$ is the Heaviside function [recall that $\Theta\left(\xi^{2}\right)=1$ for $\xi^{2}>0$ and $\Theta\left(\xi^{2}\right)=0$ otherwise], $\lambda=\mu / \varepsilon$ is the pitch angle, $\mu$ is the magnetic moment, $\sigma=v_{\|} /\left|v_{\|}\right|$. Note the relation:

$$
\langle\nabla \cdot \mathbf{A}\rangle=\frac{1}{V^{\prime}} \frac{\mathrm{d}}{\mathrm{d} s}\left[V^{\prime}\langle\mathbf{A} \cdot \nabla s\rangle\right]
$$

which has been employed in the derivation of Eq. (13). Finally, substituting the representation of the drift velocity as a sum of oscillating and averaged parts from Eq. (9) and integrating the $v_{\|} \nabla_{\|}$-term in Eq. (13) by parts (this term results from the oscillating component $v_{\|} \nabla_{\|} G$ of the radial drift velocity $\mathbf{v}_{\mathbf{d}} \cdot \nabla s$ ), one can obtain:

$$
\left\langle\int \mathrm{d}^{3} v \mathbf{v}_{\mathbf{d}} \cdot \nabla f_{a 1}\right\rangle=\frac{1}{V^{\prime}} \frac{\mathrm{d}}{\mathrm{d} s} V^{\prime} \pi \sum_{\sigma} \int\left\langle\Theta\left(\xi^{2}\right)\left[-\frac{B G}{v_{\|}} v_{\|} \nabla_{\|} f_{a 1}+\frac{B f_{a 1}}{v_{\|}} \bar{\omega}_{r}\right]\right\rangle \sigma v^{3} \mathrm{~d} v \mathrm{~d} \lambda .
$$


The perturbed distribution function appearing in Eq. (15) can be found from the kinetic equation. To the lowest order in the drift velocity (neglecting finite-orbit-width effects), the kinetic equation can be written in the form:

$$
\frac{\partial f_{a 1}}{\partial t}+v_{\|} \nabla_{\|} f_{a 1}=-\frac{e_{a} \phi^{\prime}}{T_{a}} f_{a 0}\left(\mathbf{v}_{\mathrm{d} a} \cdot \nabla s\right)
$$

The equations (12) and (16) coupled to the quasineutrality equation (4) can be considered as an initial value problem. Before solving it, note (following Xiao and Catto [6]) that in the original Rosenbluth-Hinton zonal flow problem [4], turbulent fluctuations build a charge source within a time much smaller than the bounce time but much larger than the gyroperiod. Thus, the initial zonal flow potential is produced by this turbulent charge source through classical polarization (i.e. particle departure from the guiding center). This process happens on a time scale of several ion gyration periods. After several bounce times, the initial potential is modified by the total polarization, which includes not only classical, but also the neoclassical polarization due to the guiding center departure from the flux surface. This argumentation can be translated into the initial conditions for Eqs. (12) and (16):

$$
-\frac{1}{V^{\prime}} \frac{\mathrm{d}}{\mathrm{d} s}\left[V^{\prime} \sum_{a}\left\langle\rho_{a}^{2}|\nabla s|^{2}\right\rangle \frac{n_{0 a} e_{a}^{2}}{T_{a}} \phi^{\prime}(t=0)\right]=\sum_{a} e_{a}\left\langle n_{a}(t=0)\right\rangle, \quad f_{a 1}(t=0)=0 .
$$

We start solving the kinetic equation from the observation that it is suitable to split the distribution function as follows:

$$
f_{a 1}=h-f_{a 0} \hat{\phi}^{\prime} G, \quad \hat{\phi}^{\prime}=\frac{e_{a} \phi^{\prime}}{T_{a}} .
$$

Applying the Laplace transform to the kinetic equation (16), one can write:

$$
p \mathcal{H}+v_{\|} \nabla_{\|} \mathcal{H}=f_{a 0} \hat{\Phi}^{\prime} \psi, \quad \psi=p G-\bar{\omega}_{r}
$$

Here, $\mathcal{H}(p)$ is the Laplace transform of the function $h(t), \hat{\Phi}^{\prime}(p)$ is the Laplace transform of the potential $\hat{\phi}^{\prime}(t)$ and the initial condition for the distribution function $f_{a 1}(t=0)=0$ has been written as $h(t=0)=f_{a 0} G \hat{\phi}^{\prime}(t=0)$. One can solve the kinetic equation by successive approximations assuming $\omega \ll \omega_{b}$ where $\omega$ is the characteristic frequency of the field perturbations and $\omega_{b}$ is the bounce frequency. In zeroth and first orders, one obtains:

$$
v_{\|} \nabla_{\|} \mathcal{H}^{(0)}=0, \quad p \mathcal{H}^{(0)}=f_{a 0} \hat{\Phi}^{\prime} \bar{\psi}, \quad v_{\|} \nabla_{\|} \mathcal{H}^{(1)}=f_{a 0} \hat{\Phi}^{\prime} \widetilde{\psi}
$$

Using this ordering, one can rewrite Eq. (15) in the form:

$$
\left\langle\int \mathrm{d}^{3} v \mathbf{v}_{\mathbf{d}} \cdot \nabla f_{a 1}\right\rangle=\frac{1}{V^{\prime}} \frac{\mathrm{d}}{\mathrm{d} s} V^{\prime} \pi \sum_{\sigma} \int\left\langle\Theta\left(\xi^{2}\right)\left[-\frac{B G}{v_{\|}} v_{\|} \nabla_{\|} f_{a 1}^{(1)}+\frac{B \bar{\omega}_{r}}{v_{\|}} f_{a 1}^{(0)}\right]\right\rangle \sigma v^{3} \mathrm{~d} v \mathrm{~d} \lambda .
$$


Finally, substituting the solution of the kinetic equation into the Laplace transform of Eq. (21), one can obtain:

$$
\left\langle\int \mathrm{d}^{3} v \mathbf{v}_{\mathbf{d}} \cdot \nabla \mathcal{F}_{a 1}(p)\right\rangle=\frac{1}{V^{\prime}} \frac{\mathrm{d}}{\mathrm{d} s}\left[V^{\prime} \Gamma_{a}(p)\right]
$$

with $\mathcal{F}_{a 1}(p)$ being the Laplace transform of the distribution function $f_{a 1}$ and

$$
\Gamma_{a}(p)=-2 \pi \int v^{3} \mathrm{~d} v \mathrm{~d} \lambda f_{a 0} \hat{\Phi}^{\prime} p\left[\left\langle\Theta\left(\xi^{2}\right) \frac{B}{\left|v_{\|}\right|} G \widetilde{G}\right\rangle+\left\langle\Theta\left(\xi^{2}\right) \frac{B}{\left|v_{\|}\right|} \frac{\bar{\omega}_{r}^{2}}{p^{2}}\right\rangle\right] .
$$

Here, $\widetilde{G}=G-\bar{G}$. Note that in order to derive Eq. (23), the symmetry properties in $\sigma$ of the expression under the integral have been used (only the even part survives the integration over $v_{\|}$from $-\infty$ to $\left.+\infty\right)$. For trapped particles, Eq. (23) can be rewritten in terms of the bounce averages:

$$
\Gamma_{a, t r a p}(p) \mid=-\frac{2 \pi F_{P}^{\prime}}{V^{\prime}} \frac{e_{a} \Phi^{\prime}}{T_{a}} \int \mathrm{d} v \mathrm{~d} \lambda f_{a 0} v^{3} \oint \mathrm{d} \alpha \sum_{n}\left(\frac{p \hat{\tau}_{b}}{2}\left[\overline{G^{2}}+\frac{\bar{\omega}_{r}^{2}}{p^{2}}\right]\right)_{n} .
$$

Here, the sum is taken over all possible sorts of trapped particles (toroidally-trapped particles, helically-trapped particles, etc) for a given field line labeled by $\alpha=\varphi-q(s) \theta$. Note that along each such field line, there are a number of magnetic wells where particles with large enough pitch angles are trapped. The orbit averages correspond to each such well, labeled by the number $n$ (see Figs. 1 and 2). In Eq. (24), we have introduced a new quantity (the bounce time) $\hat{\tau}_{b}=\oint(B \sqrt{g}) /\left(F_{P}^{\prime} v_{\|}\right) \mathrm{d} \theta$ with the integral taken along the field line (back and forth) between the reflecting points. Note that the property $\bar{G}=0$ for trapped particles has been used in Eq. (24).

For passing particles, Eq. (23) can be written as follows:

$$
\Gamma_{a, p a s s}(p)=-2 \pi \int v^{3} \mathrm{~d} v \mathrm{~d} \lambda f_{a 0} \frac{e_{a} \Phi^{\prime}}{T_{a}} p \int \mathrm{d} \lambda\left[\left\langle\frac{B}{\left|v_{\|}\right|} G^{2}\right\rangle-\left\langle\frac{B}{\left|v_{\|}\right|}\right\rangle^{-1}\left\langle\frac{B}{\left|v_{\|}\right|} G\right\rangle^{2}\right]
$$

This expression can be rewritten in the same form as Eq. (24) for $\Gamma_{a, t r a p}$ taking into account that for the passing particles the orbit average is defined according to Eq. (8) and $\bar{\omega}_{r}=0$.

Applying the Laplace transform to the continuity equation (12), one finds:

$$
p \mathcal{N}_{a}(p)-\left\langle n_{a}(t=0)\right\rangle+\frac{1}{V^{\prime}} \frac{\mathrm{d}}{\mathrm{d} s}\left[V^{\prime} \Gamma_{a}(p)\right]=0
$$

where $\mathcal{N}_{a}(p)$ is the Laplace transform of $\left\langle n_{a}(t)\right\rangle$ and $\left\langle n_{a}(t=0)\right\rangle$ is related to the electrostatic potential through Eq. (17). Substituting the densities $\mathcal{N}_{a}$ into the the Laplace-transform of 
the quasineutrality equation (4) and integrating over $s$, one can obtain the relation between the residual potential and the initial one as follows:

$$
\Phi^{\prime}(p)=\left[1+\Lambda(p) / \Lambda_{0}\right]^{-1} \frac{\phi^{\prime}(t=0)}{p}
$$

where the classical polarization is given by the quantity $\Lambda_{0}$ :

$$
\Lambda_{0}=\sum_{a} \frac{n_{0 a} e_{a}^{2}}{T_{a}}\left\langle\rho_{a}^{2}|\nabla s|^{2}\right\rangle
$$

The neoclassical polarization and the effect of the radial bounce-averaged drift motion are contained in the quantity:

$$
\begin{aligned}
\Lambda(p)= & \underbrace{2 \pi \sum_{a} \int \mathrm{d} v \mathrm{~d} \lambda \frac{e_{a}^{2} f_{a 0}}{T_{a}} v^{3}\left[\left\langle\frac{B}{\left|v_{\|}\right|} G^{2}\right\rangle-\left\langle\frac{B}{\left|v_{\|}\right|}\right\rangle^{-1}\left\langle\frac{B}{\left|v_{\|}\right|} G\right\rangle^{2}\right]}_{\text {passing particles }}+ \\
& +\underbrace{\frac{2 \pi F_{P}^{\prime}}{V^{\prime}} \sum_{a} \int \mathrm{d} v \mathrm{~d} \lambda \frac{e_{a}^{2} f_{a 0}}{T_{a}} v^{3} \oint \mathrm{d} \alpha \sum_{n}\left(\frac{\hat{\tau}_{b}}{2}\left[\overline{G^{2}}+\frac{\bar{\omega}_{r}^{2}}{p^{2}}\right]\right)_{n, a}}_{\text {trapped particles }} .
\end{aligned}
$$

Taking the integral over the velocity in Eq. (29) and transforming Eq. (27) back into the time domain (recall that the inverse Laplace transform is $\mathcal{L}^{-1}\left[p /\left(p^{2}+a^{2}\right), t\right]=\cos (a t)$, see Ref. [16]), one can write the residual zonal flow in the form:

$$
\frac{\phi^{\prime}(t)}{\phi^{\prime}(t=0)}=\left(1+\Lambda_{1} / \Lambda_{0}\right)^{-1} \cos (\Omega t), \quad \Omega=\sqrt{\frac{\Lambda_{2}}{\Lambda_{0}+\Lambda_{1}}} .
$$

Here, the neoclassical polarization enters through the quantity $\Lambda_{1}$ :

$$
\begin{aligned}
\Lambda_{1}=\frac{3}{2} \sum_{a} \frac{n_{0 a} e_{a}^{2}}{T_{a}} \int \mathrm{d} \lambda & {[\underbrace{\left\langle\left\langle\frac{B}{\xi} G_{\mathrm{th}}^{2}\right\rangle-\left\langle\frac{B}{\xi}\right\rangle^{-1}\left\langle\frac{B}{\xi} G_{\mathrm{th}}\right\rangle^{2}\right)}_{\text {passing particles }}+} \\
& +\underbrace{\frac{F_{P}^{\prime} v_{\mathrm{th} a}}{V^{\prime}} \oint \mathrm{d} \alpha \sum_{n}\left(\frac{\hat{\tau}_{b \mathrm{th}}}{2} \overline{G_{\mathrm{th}}^{2}}\right)_{n, a}}_{\text {trapped particles }}]
\end{aligned}
$$

and the frequency of the zonal-flow mode is given by the quantity $\Lambda_{2}$ :

$$
\Lambda_{2}=\underbrace{\frac{15}{2} \sum_{a} \frac{n_{0 a} e_{a}^{2}}{T_{a}} \frac{F_{P}^{\prime} v_{\text {tha }}}{V^{\prime}} \int \mathrm{d} \lambda \oint \mathrm{d} \alpha \sum_{n}\left(\frac{\hat{\tau}_{b \mathrm{th}}}{2} \bar{\omega}_{r \mathrm{th}}^{2}\right)_{n, a}}_{\text {trapped particles }}
$$


with the first sum taken over particle species (ions, impurities and electrons) and the second sum (in the trapped-particle term) taken over the groups of particles (banana-trapped, locally-trapped etc). Note that only trapped particles contribute to the quantity $\Lambda_{2}$. The index th in Eqs. (31) and (32) means that all energy-dependent quantities are computed according to their definitions but using the thermal velocity $v_{\text {tha }}=\sqrt{T_{a} / m_{a}}$ instead of $v$. Note that $v_{\text {tha }}$ is used solely as a normalization constant to keep proper dimensions of various quantities and that the integrals over the velocity in Eq. (29) have been computed exactly [the coefficients $3 / 2$ and 15/2 in Eqs. (31) and (32) result from this computation].

\section{SOLUTION OF THE MAGNETIC DIFFERENTIAL EQUATION}

In this paragraph we solve the magnetic differential equation (10) for the function $G$. For passing particles the equation is solved by Fourier transform with respect to the toroidal and poloidal angles. For trapped particles, the periodic character of the particle motion is employed to solve Eq. (10) using a bounce-harmonic expansion.

\section{A. Passing particles}

For passing particles $\bar{\omega}_{r}=0$ so that the magnetic differential equation (10) for the quantity $G$ takes the form:

$$
v_{\|} \nabla_{\|} G=\omega_{r}
$$

In Boozer coordinates, this equation can be rewritten as follows:

$$
\left(F_{P}^{\prime} \partial_{\theta}+F_{T}^{\prime} \partial_{\varphi}\right) G=\left(I \partial_{\theta}-J \partial_{\varphi}\right) \rho_{\|}
$$

Assuming a non-resonant flux surface, one can solve this equation by applying a Fourier transform with respect to the poloidal and toroidal angles:

$$
\left.G\right|_{\text {passing }}=G_{00}+\sum_{m, n \neq 0} \frac{m I-n J}{m F_{P}^{\prime}+n F_{T}^{\prime}} \rho_{\| m n} \exp (i m \theta+i n \varphi) .
$$

Note that the final result Eq. (31) does not depend on the particular choice of $G_{00}$ (the corresponding contribution in $\Lambda_{1}$ vanishes). Thus, choosing $G_{00}=I \rho_{\| 00} / F_{P}^{\prime}$, one can write the solution in the form:

$$
\left.G\right|_{\text {passing }}=\frac{I}{F_{P}^{\prime}} \rho_{\|}-\sum_{m, n \neq 0} \frac{q n}{m+q n}\left(1+\frac{J}{q I}\right) \frac{I}{F_{P}^{\prime}} \rho_{\| m n} \exp (i m \theta+i n \varphi) .
$$


In the tokamak case, the second term in Eq. (36) vanishes because only components with $n=0$ contribute. Thus, $G$ takes the form which coincides with the conventional tokamak expression (see Ref. [6]). In our numerical calculations (see below), we neglect the second term in Eq. (36) also in stellarator geometry. This approximation makes only a small difference to our results.

\section{B. Trapped particles}

Before solving the magnetic differential equation for trapped particles, it is instructive to rewrite the radial drift velocity as follows:

$$
\omega_{r}=\mathbf{v}_{\mathbf{d}} \cdot \nabla s=\tau_{b}^{-1}\left[\frac{I}{F_{P}^{\prime}}\left(\frac{\partial \rho_{\|}}{\partial \theta}\right)_{\alpha}-\frac{B^{2} \sqrt{g}}{F_{P}^{\prime 2}}\left(\frac{\partial \rho_{\|}}{\partial \alpha}\right)_{\theta}\right]=\omega_{r \theta}-\omega_{r \alpha} .
$$

Here, $\tau_{b}=(B \sqrt{g}) /\left(F_{P}^{\prime} v_{\|}\right)$and $\alpha=\varphi-q(s) \theta$ is the field line label [i.e. the derivative with respect to $\theta$ is taken in Eq. (37) along a fixed field line]. Note that $B^{2} \sqrt{g}$ is a function of $s$ only (recall that we use Boozer coordinates). Using the notation introduced in Eq. (37), one can write the magnetic differential equation (10) for trapped particles in the form:

$$
\tau_{b}^{-1} \frac{\partial}{\partial \theta}\left(\frac{I}{F_{P}^{\prime}} \rho_{\|}-G\right)_{\alpha=\mathrm{const}}=\widetilde{\omega}_{r \alpha}, \quad \widetilde{\omega}_{r \alpha}=\omega_{r \alpha}-\bar{\omega}_{r \alpha}, \bar{\omega}_{r \alpha}=\bar{\omega}_{r} .
$$

Let us introduce the "effective time" variable according to the following relation:

$$
\tau=\left\{\begin{aligned}
\sigma(\tau) \int_{\theta_{1}}^{\theta} \tau_{b} \mathrm{~d} \theta & : \quad 0 \leq \tau \leq \hat{\tau}_{b} / 2 \\
\hat{\tau}_{b} / 2+\sigma(\tau) \int_{\theta_{2}}^{\theta} \tau_{b} \mathrm{~d} \theta & : \quad \hat{\tau}_{b} / 2 \leq \tau \leq \hat{\tau}_{b}
\end{aligned}\right.
$$

Here, $\left(\theta_{1}, \theta_{2}\right)$ are the reflecting points for a given class of trapped particles on a given field line and $\hat{\tau}_{b}=\left|\oint \tau_{b} \mathrm{~d} \theta\right|$ is the bounce time. In terms of $\tau$, one can write Eq. (38) as follows:

$$
\frac{\partial}{\partial \tau}\left(\frac{I}{F_{P}^{\prime}} \rho_{\|}-G\right)=\sigma(\tau) \widetilde{\omega}_{r \alpha}(\tau)
$$

Introducing the notation $I \rho_{\|} / F_{P}^{\prime}=\sigma \hat{\rho}, G=\sigma \hat{G}$, the bounce frequency $\hat{\omega}_{b}=2 \pi / \hat{\tau}_{b}$ and the new dimensionless "time" $\zeta=\hat{\omega}_{b} \tau(0 \leq \zeta \leq 2 \pi)$, we can write Eq. (40) in the form:

$$
(\hat{\rho}-\hat{G}) \frac{\partial \sigma}{\partial \zeta}+\sigma \frac{\partial}{\partial \zeta}(\hat{\rho}-\hat{G})=\sigma \widetilde{\omega}_{r \alpha} / \hat{\omega}_{b} .
$$

Note that the coefficient before $\partial \sigma / \partial \zeta$ must vanish at $\zeta=\pi n(n=0,1,2)$ where the function $\sigma(\zeta)$ is not continuous. Hence, the following relation holds:

$$
\hat{G}(\pi n)=\hat{\rho}(\pi n), \quad n=0,1,2 .
$$


Since the motion of trapped particles is periodic in $\zeta$ (with the period $2 \pi$ ), one can apply the Fourier transform in $\zeta$ (the so-called bounce-harmonic expansion):

$$
f(\zeta)=\sum_{l=-\infty}^{+\infty} f_{l} \exp (-i l \zeta), \quad f_{l}=\frac{1}{2 \pi} \int_{0}^{2 \pi} f(\zeta) \exp (i l \zeta) \mathrm{d} \zeta .
$$

Note that the zero bounce harmonic coincides with the bounce average $\bar{f}=f_{0}$. Using this relation, one can write the following expressions:

$$
\widetilde{\omega}_{r \alpha}(\zeta)=\sum_{l \neq 0} \omega_{r l} \exp (-i l \zeta), \omega_{r l}=\frac{1}{2 \pi} \int_{0}^{2 \pi} \omega_{r \alpha}(\zeta) \exp (i l \zeta) \mathrm{d} \zeta .
$$

Note that the sum over $l$ in Eq. (44) does not include the zero bounce-harmonic coefficient $\omega_{r 0}$ because $\widetilde{\omega}_{r \alpha}=\omega_{r \alpha}-\bar{\omega}_{r \alpha}$. One can express the "regularity condition" Eq. (42) in terms of the bounce-harmonic coefficients as follows:

$$
\hat{G}_{0}=\hat{\rho}_{0} \quad \text { and } \quad \hat{G}_{l}+\hat{G}_{-l}=\hat{\rho}_{l}+\hat{\rho}_{-l}, \quad l \neq 0 .
$$

The coefficients $G_{l}$ for $l \neq 0$ can be found from the solution of the magnetic differential equation which now takes the form:

$$
\frac{\partial}{\partial \zeta}(\hat{\rho}-\hat{G})=\widetilde{\omega}_{r \alpha} / \hat{\omega}_{b} \Longleftrightarrow-i l\left(\hat{\rho}_{l}-\hat{G}_{l}\right)=\omega_{r l} / \hat{\omega}_{b}, \quad l \neq 0 .
$$

Finally, one can write the coefficients $G_{l}$ for $l \neq 0$ as follows:

$$
\hat{G}_{l}=\hat{\rho}_{l}-i \hat{a}_{l}, \quad \hat{a}_{l}=\omega_{r l} /\left(l \hat{\omega}_{b}\right) .
$$

Now, one can use the Parseval's Theorem to compute the quantity $\overline{G^{2}}$ needed in Eq. (31):

$$
\overline{G^{2}}=\frac{1}{2 \pi} \int_{0}^{2 \pi}|G(\zeta)|^{2} \mathrm{~d} \zeta=\sum_{l=-\infty}^{+\infty}\left|\hat{G}_{l}\right|^{2}=\hat{\rho}_{0}^{2}+\sum_{l \neq 0}\left(\hat{\rho}_{l}^{2}+\hat{a}_{l}^{2}\right) .
$$

Here, we have used the relation $G^{2}=\hat{G}^{2}$ and the "regularity condition" $\hat{G}_{0}=\hat{\rho}_{0}$. Note that in an axisymmetric geometry the coefficient $\hat{a}_{l}=0$ so that the tokamak result can be recovered.

One can show that $\hat{\rho}_{l}=\hat{\rho}_{-l}$ and $\hat{a}_{l}=-\hat{a}_{-l}$ so that the "regularity condition" Eq. (45) holds for the coefficients with $l \neq 0$. One can prove these properties of the coefficients $\hat{\rho}_{l}$ and $\hat{a}_{l}$ using the following symmetry relations:

$$
\hat{\rho}(\zeta)=\hat{\rho}(2 \pi-\zeta), \omega_{r \alpha}(\zeta)=\omega_{r \alpha}(2 \pi-\zeta)
$$


Note that the "times" $\zeta$ and $2 \pi-\zeta$ correspond to the same point in the space on the back and forth motion of a trapped particle along the field line. Substituting Eq. (49) into the definition of the bounce-harmonic coefficients Eq. (43), one can show that

$$
\hat{\rho}_{l}=\frac{1}{\pi} \int_{0}^{\pi} \hat{\rho}(\zeta) \cos (l \zeta) \mathrm{d} \zeta, \quad \omega_{r l}=\frac{1}{\pi} \int_{0}^{\pi} \omega_{r \alpha}(\zeta) \cos (l \zeta) \mathrm{d} \zeta, \quad \hat{a}_{l}=\frac{1}{\pi} \int_{0}^{\pi} \frac{\omega_{r \alpha}(\zeta)}{\hat{\omega}_{b}} \frac{\cos (l \zeta)}{l} \mathrm{~d} \zeta
$$

Thus, the "regularity condition" Eq. (45) for $l \neq 0$ is a direct consequence of the symmetry relations Eq. (49). Using Eq. (50), one can write the expression for $\overline{G^{2}}$ as follows:

$$
\overline{G^{2}}=\hat{\rho}_{0}^{2}+2 \sum_{l>0}\left(\hat{\rho}_{l}^{2}+\hat{a}_{l}^{2}\right)
$$

\section{NUMERICAL ALGORITHM}

In Sec. III, we have expressed the Rosenbluth-Hinton residual flow amplitude and its frequency in terms of some flux-surface averages (passing particles) and bounce averages (trapped particles) [see Eqs. (30), (31) and (32)]. The quantity G (see Sec. IV) resulting from the solution of a magnetic differential equation (10) is expressed for trapped particles in terms of the bounce-harmonic coefficients Eq. (51). These flux-surface averages, bounce averages and bounce-harmonic coefficients are computed numerically using equilibria obtained from the VMEC code $[17,18]$ and transformed to Boozer coordinates $[2,15]$.

While the flux surface averages can be calculated easily, more effort is necessary for the bounce averages and the bounce-harmonic coefficients of the trapped particles. For given flux surface $s$, field line $\alpha$ and pitch angle $\lambda$, all possible classes of trapped particles can be identified solving $1-\lambda B\left(s, \theta_{i}, \alpha\right)=0$. The roots of this equation $\theta_{i}, i=1,2, \ldots$ define all possible bounce points on the flux surface and, as a consequence, all possible sorts of trapped particles. These bounce points define the limits of integration in the calculation of the orbit averages and the bounce-harmonic coefficients.

The kinetic part of the CAS3D-K [19] code package has been used to cover the phase space $\left(s, \alpha, \theta_{i}, \lambda\right)$ with orbits and associated reflection points for the trapped particles (see Fig. 1). This code solves the resulting tedious root finding problem described before and groups similar orbits together thus allowing a precise sampling of the phase space with orbits (see Fig. 2). The details of the code will be published elsewhere. We will give just the resolution parameters which have been used: $3 \times 4$ Gaussian points (Gauss-Legendre quadrature) for 
the trapping parameter $\lambda$, 32 Gaussian spaced field line labels (Gauss-Legendre quadrature) per particle group and at least 70 Gaussian points (Gauss-Chebychev quadrature) per field line. For the calculation of the bounce averages 30 Fourier components of $|B(s, \theta, \alpha)|$ have been maintained.

\section{DISCUSSION AND CONCLUSIONS}

In the previous sections we have seen that the linear response of a non-axisymmetric plasma to an applied radial electric field generally has an oscillatory character. Physically, this may be understood as follows. Following Hinton and Rosenbluth [4, 5], we have been considering how the potential evolves in response to a radial voltage perturbation at $t=0$. In tokamak geometry, after a few ion bounce times, the radial electric field in the plasma is smaller than the initially applied field because the plasma is polarizable: the ion banana orbits move radially in such a way that they shield much of the applied voltage. The plasma thus acts as a capacitor, and the radial voltage is related to the current as

$$
i(t)=C \frac{d u}{d t}
$$

In stellarators, there is also an additional effect due to the presence of locally trapped particles with net radial drift. Some of these particles drift radially inward while others drift outward, but there is no net current (on a flux-surface average) if the distribution function is the equilibrium Maxwellian - the inward and outward currents then cancel. However, if the radial electric field is applied for some finite time, then the distribution function starts to depart from a Maxwellian. The outward drifting ions gain energy (if the radial electric field points outward) and the inward drifting ones lose energy, and vice versa for the electrons. Since the drift velocity is proportional to $v^{2}$, the speed of the outward drifting ions increases with time, the inward drifting ones get slower, and a net current arises that is proportional to the time integral of the voltage. Thus

$$
i(t)=L^{-1} \int_{0}^{t} u\left(t^{\prime}\right) d t^{\prime}+C \frac{d u}{d t},
$$

so the plasma behaves like an LC-circuit and oscillates at a frequency $\Omega=(L C)^{-1 / 2}$. It is beyond the scope of the present paper to consider the effect of collisions in detail, but we note that these also produce a radial current. A stellarator is in general not automatically 
ambipolar, and the equilibrium radial electric field is set by the requirement that the neoclassical particle fluxes of ions and electrons should be equal. In the vicinity of this equilibrium, a radial current arises that is proportional to the departure from the ambipolar electric field [12]. On time scales longer than the (electron) collision time, neoclassical transport thus provides a resistor in the LC-circuit, which leads to damping of the zonal flow oscillations. The turbulence introduces a stochastic generator into our circuit.

An important question raised already in Refs. $[9,12]$ is the link between the neoclassicaltransport optimization and the reduction of the anomalous transport. The discussion in this respect has been triggered by experimental results from the Large Helical Device [13] (LHD). It has been observed that not only the neoclassical but also anomalous transport is reduced by the inward shift of the magnetic axis in LHD. The inward shift decreases the radial drift of helically-trapped particles but it increases the unfavorable magnetic curvature destabilizing pressure-gradient-driven instabilities such as the ITG mode (see Ref. [8] and the references therein), which should lead to a higher level of the anomalous transport. On the other hand, in Refs. [9, 12], it is suggested that the properties of zonal flows are more favorable in the drift-optimized configurations. The reason is that the residual RosenbluthHinton level is larger, which leads to a more effective suppression of the turbulence in the inward-shifted configuration. The zonal flow oscillations found in this paper are however not considered in Refs. [9, 12].

Using our numerical approach, we have studied the effect of drift optimization on zonal flow parameters (its amplitude and eigenfrequency). In Figs. 3 and 4 we compare the socalled standard LHD configuration with the inward-shifted LHD configuration and observe a substantially smaller frequency in the inward-shifted configuration. At the same time, the amplitude of the zonal flow is fairly similar over most of the plasma volume, suggesting that the main effect of the drift optimization is the reduction of the frequency and not an increase of the residual zonal-flow amplitude.

In Figs. 5 and 6 we compare the Wendelstein 7-X configuration (an optimized stellarator, see Ref. [14]) with the "equivalent" $l=2$ classical stellarator (i.e. the classical stellarator having the same minor and major radii, rotation transform and the magnetic field on axis). Again, the main effect of the optimization is a substantial reduction of the zonal-flow eigenfrequency whereas the residual-flow amplitude is actually larger in the non-optimized case. Of course, we should note that the W7-X and the "equivalent" $l=2$ stellarator are 
much more different configurations than the standard and the inward-shifted ones in LHD. Nevertheless, this example is a good illustration of the role of the drift optimization in the zonal-flow dynamics in stellarators. This behavior can be deduced from the formal expressions we have derived. Note that the zonal-flow eigenfrequency $\Omega \sim \sqrt{\Lambda_{2}}$ is a measure of the bounce-averaged radial drifts of the particles [see Eq. (32)]. Clearly, reduction of the radial drifts makes $\Omega$ smaller.

The role of electrons is different from that in tokamaks. Having the same bounce-averaged radial-drift velocities, electrons make a contribution to the zonal-flow eigenfrequency comparable to that of ions. At the same time, the contribution of the electrons to the neoclassical polarization remains negligibly small (proportional to the mass ratio, as it is the case in tokamaks).

Finally, we can speculate that the reduction of the anomalous transport in the driftoptimized configuration occurs because the zonal-flow eigenfrequency is directly affected by the drift reduction. Note that the zonal flow is constantly produced (in a non-coherent way) by the turbulence (recall the "stochastic generator" in our LC-circuit). Thus, it is instructive to estimate response of our system to a noise source. Following Ref. [5], the mean square potential can be written as

$$
\left\langle\mid\left\langle\left.\phi_{k}(t)\right|^{2}\right\rangle\right\rangle=\int_{0}^{t} \mathrm{~d} t_{1} \int_{0}^{t_{1}} \mathrm{~d} t_{2}\left\langle\left\langle R_{k}\left(t_{1}\right) R_{k}\left(t_{2}\right)\right\rangle\right\rangle K_{k}\left(t_{1}\right) K_{k}\left(t_{2}\right),
$$

where the double brackets indicate a statistical average, the kernel $K_{k}(t)=\phi_{k}(t) / \phi_{k}(t=0)$ results from the linear theory and the noise source correlation function is modelled according to the equation:

$$
\left\langle\left\langle R_{k}\left(t_{1}\right) R_{k}\left(t_{2}\right)\right\rangle\right\rangle=\left\langle\left\langle\left|R_{k}\right|^{2}\right\rangle\right\rangle \exp \left(-\tau^{2} / \tau_{c}^{2}\right)
$$

with $\tau=t_{1}-t_{2}$ and $\tau_{c}$ being the correlation time. Substituting $K_{k}(t)=A_{R} \cos \Omega t$ in Eq. (54), one finds that the mean square potential can be estimated for $t \gg\left(1 / \Omega, \tau_{c}\right)$ as

$$
\frac{\left\langle\left\langle\left|\phi_{k}(t)\right|^{2}\right\rangle\right\rangle}{\left\langle\left\langle\left|R_{k}\right|^{2}\right\rangle\right\rangle \tau_{c}^{2}} \sim A_{R}^{2} \exp \left(-\frac{\Omega^{2} \tau_{c}^{2}}{4}\right) .
$$

In Eq. (56), we have neglected the term corresponding to the GAM oscillations because of their large frequency $\omega_{f} \tau_{c} \gg 1$ leading to a near cancellation of this contribution into the mean square potential [5]. One sees that the mean square potential can be substantially reduced if the zonal-flow frequency is large enough so that $\Omega \tau_{c}>1$ is satisfied. This can 
be referred to as a "phase-mixing mechanism" of the collisionless zonal-flow damping. In stellarators, this mechanism acts in addition to the usual Rosenbluth-Hinton shielding due to neoclassical polarization of the plasma [represented in Eq. (56) through the quantity $A_{R}$ ].

In Ref. [20], it has been suggested that the zonal flow damping can be further reduced by the background (e.g. neoclassical) electric field $\mathbf{E}_{\mathbf{0}}=-\nabla \Phi_{0}$. The reason for that is the reduction of the average radial displacement of the helically-trapped particles by the corresponding $\mathbf{E}_{\mathbf{0}} \times \mathbf{B}$ drift which enters the kinetic equation (20) as follows (here $\alpha$ is the field-line label):

$$
\left(p+\Omega_{E} \frac{\partial}{\partial \alpha}\right) \mathcal{H}^{(0)}=f_{a 0} \hat{\Phi}^{\prime} \bar{\psi}, \quad \psi=\left(p+\Omega_{E} \frac{\partial}{\partial \alpha}\right) G-\bar{\omega}_{r}, \quad \Omega_{E}=\frac{1}{F_{P}^{\prime}} \frac{\mathrm{d} \Phi_{0}}{\mathrm{~d} s} .
$$

Clearly, solution of this equation will lead to a different time dependence of the residual potential $\phi$ than the solution of Eq. (20). As an example, we consider a poloidally symmetric, large-aspect-ratio stellarator with circular cross section, where $\bar{\omega}_{r}=\Omega_{r}(\psi) \sin \alpha, \partial G / \partial \alpha=0$ and $\partial \hat{\tau}_{b} / \partial \alpha=0$. In this case, one finds the following expression for the residual flow:

$$
\begin{aligned}
& \frac{\phi^{\prime}(t)}{\phi^{\prime}(t=0)}=A_{R}\left[\frac{\Omega_{E}^{2}}{\Omega^{2}}+\left(1-\frac{\Omega_{E}^{2}}{\Omega^{2}}\right) \cos \Omega t\right] \\
& \Omega=\sqrt{\Omega_{Z F}^{2}+\Omega_{E}^{2}}, \quad \Omega_{Z F}=\sqrt{\frac{\Lambda_{2}}{\Lambda_{0}+\Lambda_{1}}}, \quad A_{R}=\frac{1}{1+\Lambda_{1} / \Lambda_{0}}
\end{aligned}
$$

One sees that a zero-frequency component appears in the plasma response whereas the amplitude of the oscillatory response is decreased by finite $\Omega_{E}$. At least in this simple case, the phase-mixing mechanism of the zonal-flow collisionless damping is reduced by the background electric field. This subject deserves a more detailed study in future.

Note that if the radial scale of the electrostatic field perturbation is short enough, the finite-orbit-width term $\mathbf{v}_{\mathbf{d a}} \cdot \nabla f_{a 1}$ neglected in Eq. (16) may become important, too. Formally, this is a small term, since it is assumed that the radial wavelength of the zonal flow exceeds the ion gyroradius. However, in Ref. [9], it was shown that this term can lead to a damping of the zonal flow due to vanishing of the non-adiabatic response of the helicallytrapped particles at the times $t \geq \tau_{r} \sim L_{r} / V_{d r}$ where $L_{r}$ is a characteristic radial length of the electrostatic field perturbation and $V_{d r}$ is the bounce-averaged drift velocity. Note, however, that for the long-wavelength part of the electrostatic field, the time $\tau_{r}$ is large whereas for the short-wavelength part it can become comparable to the zonal flow frequency. Thus, one can expect that the collisionless dynamics of the long-wavelength part of the electrostatic field is mainly controlled by the oscillations found in this work whilst the dynamics 
of the short-wavelength part is dominated by the finite-orbit-width mechanism described in Ref. [9].

Another problem which has not been considered in this paper is the role of collisions. In general, collisions introduce a damping mechanism for the zonal flow [5, 21]. For example, in the limit $\nu_{e} \gg \Omega$ (here, $\nu_{e}$ the electron collision frequency and $\Omega$ the zonal flow frequency) one can show that electron collisions produce exponential damping of the zonal flow:

$$
\frac{\phi(t)}{\phi(t=0)}=A_{R} \exp \left(-\gamma_{Z F} t\right) \cos \Omega t
$$

Furthermore, in this case the electrons are omitted from the sum in Eq. (32) and do not contribute to the frequency $\Omega$. The damping rate $\gamma_{Z F}$ is defined by the collisional flux of the trapped electrons. This flux can be shown to be inversely proportional to the collision frequency. As a consequence, one can show that $\gamma_{Z F} / \Omega \sim \Omega / \nu_{e} \ll 1$. A detailed calculation will be presented elsewhere.

\section{ACKNOWLEDGMENTS}

We acknowledge discussions with H. Sugama. We thank Yu. Turkin and J. Geiger for their help on the numerical part of the work. We appreciate comments of R. Kleiber and J. Nührenberg on the physical interpretation of our results. 


\section{REFERENCES}

[1] H. E. Mynick, Phys. Plasmas 13, 058102 (2006).

[2] J. Nührenberg and R. Zille, Phys. Lett. A 129, 113 (1988).

[3] P. H. Diamond, S.-I. Itoh, K. Itoh, and T. S. Hahm, Plasma Phys. Controlled Fusion 47, R35 (2005).

[4] M. Rosenbluth and F. Hinton, Phys. Rev. Lett. 80, 724 (1998).

[5] F. Hinton and M. Rosenbluth, Plasma Phys. Controlled Fusion 41, A653 (1999).

[6] Y. Xiao and P. Catto, Phys. Plasmas 13, 082307 (2006).

[7] Y. Xiao, P. Catto, and W. Dorland, Phys. Plasmas 14, 055910 (2007).

[8] H. Sugama and T.-H. Watanabe, Phys. Rev. Lett. 94, 115001 (2005).

[9] H. Sugama and T.-H. Watanabe, Phys. Plasmas 13, 012501 (2006).

[10] H. Sugama, T.-H. Watanabe, and W. Horton, Phys. Plasmas 14, 022502 (2007).

[11] S. Ferrando-Margalet, H. Sugama, and T.-H. Watanabe, Phys. Plasmas 14, 122505 (2007).

[12] H. E. Mynick and A. H. Boozer, Phys. Plasmas 14, 072507 (2007).

[13] O. Motojima, N. Ohyabu, A. Komori, O. Kaneko, H. Yamada, K. Kawahata, Y. Nakamura, K. Ida, T. Akiyama, N. Ashikawa, W. A. Cooper, A. Ejiri, M. Emoto, N. Ezumi, H. Funaba, A. Fukuyama, P. Goncharov, M. Goto, H. Idei, K. Ikeda, S. Inagaki, M. Isobe, S. Kado, H. Kawazome, K. Khlopenkov, T. Kobuchi, K. Kondo, A. Kostrioukov, S. Kubo, R. Kumazawa, Y. Liang, J.F. Lyon, A. Mase, S. Masuzaki, T. Minami, J. Miyazawa, T. Morisaki, S. Morita, S. Murakami, S. Muto, T. Mutoh, K. Nagaoka, Y. Nagayama, N. Nakajima, K. Nakamura, H. Nakanishi, K. Narihara, Y. Narushima, K. Nishimura, N. Nishino, N. Noda, T. Notake, H. Nozato, S. Ohdachi, Y. Oka, H. Okada, S. Okamura, M. Osakabe, T. Ozaki, B. J. Peterson, A. Sagara, T. Saida, K. Saito, S. Sakakibara, M. Sakamoto, R. Sakamoto, M. Sasao, K. Sato, M. Sato, T. Seki, T. Shimozuma, M. Shoji, H. Suzuki, Y. Takeiri, N. Takeuchi, N. Tamura, K. Tanaka, M. Y. Tanaka, Y. Teramachi, K. Toi, T. Tokuzawa, Y. Tomota, Y. Torii, K. Tsumori, K.Y. Watanabe, T. Watari, Y. Xu, I. Yamada, S. Yamamoto, T. Yamamoto, M. Yokoyama, S. Yoshimura, Y. Yoshimura, M. Yoshinuma, N. Asakura, T. Fujita, T. Fukuda, T. Hatae, S. Higashijima, A. Isayama, Y. Kamada, H. Kubo, Y. Kusama, Y. Miura, T. Nakano, H. Ninomiya, T. Oikawa, N. Oyama, Y. Sakamoto, K. Shinohara, 
T. Suzuki, H. Takenaga, K. Ushigusa, T. Hino, M. Ichimura, Y. Takase, F. Sano, H. Zushi, T. Satow, S. Imagawa, T. Mito, I. Ohtake, T. Uda, K. Itoh, K. Ohkubo, S. Sudo, K. Yamazaki, K. Matsuoka, Y. Hamada and M. Fujiwara, Nucl. Fusion 43, 1674 (2003).

[14] G. Grieger, C. D. Beidler, H. Maassberg, E. Harmeyer, F. Herrnegger, J. Junker, J. Kisslinger, W. Lotz, P. Merkel, J. Nührenberg, F. Rau, J. Sapper, A. Schlueter, F. Sardei, and H. Wobig, in Proceedings of the 13th International Conference on Plasma Physics and Controlled Nuclear Fusion Research (International Atomic Energy Agency, Vienna, 1991), Vol. 3, p. 525.

[15] A. Boozer, Phys. Fluids 25, 520 (1982).

[16] I. S. Gradshteyn and I. M. Ryzhik, Table of integrals, series and products (Academic Press, New York, 1980).

[17] S. P. Hirshman and D. K. Lee, Comp. Phys. Commun. 39, 161 (1986).

[18] S. P. Hirshman, W. I. van Rij, and P. Merkel, Comp. Phys. Commun. 43, 143 (1986).

[19] A. Könies, in Proceedings of the 7th IAEA Technical Committee Meeting on Energetic Particles in Magnetic Confinement Systems (Chalmers University of Technology, Göteborg, Sweden, 2001), CD-ROM.

[20] H. Sugama, T.-H. Watanabe, and S. Ferrando-Margalet, in Proceedings of the Joint Conference of 17th International Toki Conference on Physics of Flows and Turbulence in Plasmas and 16th International Stellarator/Heliotron Workshop (Toki, Gifu, Japan, 2007), CD-ROM.

[21] Y. Xiao, P. Catto, and K. Molvig, Phys. Plasmas 14, 032302 (2007). 


\section{Figure captions}

Fig. 1: modulus of $B$ for the W7-X standard case in Boozer coordinates at $s=0.48$. A value of the trapping parameter $\lambda$ defines the intersecting plane. The particles move (within the approximation used) on field lines on this plane. Some of the field lines are sketched.

Fig. 2 (Color online): groups of particle orbits for $\lambda=2.4546$ and $s=0.48$ for the W7-X standard case in Boozer coordinates. The numbers indicate the number of periods the different particle groups cross until they reach the reflection point.

Fig. 3 (Color online): the residual zonal-flow frequency. The inward-shifted LHD equilibrium vs. the standard LHD equilibrium. The ion and electron temperatures $T_{i}=T_{e}=5 \mathrm{keV}$ (flat profiles), Hydrogen ions.

Fig. 4 (Color online): the residual zonal-flow amplitude. The same equilibria and parameters as in Fig. 3.

Fig. 5 (Color online): the residual zonal-flow frequency. A standard Wendelstein 7-X equilibrium vs. the "equivalent" $l=2$ classical stellarator equilibrium. The ion and electron temperatures $T_{i}=T_{e}=5 \mathrm{keV}$ (flat profiles), Hydrogen ions.

Fig. 6 (Color online): the residual zonal-flow amplitude. The same equilibria and parameters as in Fig. 5. 


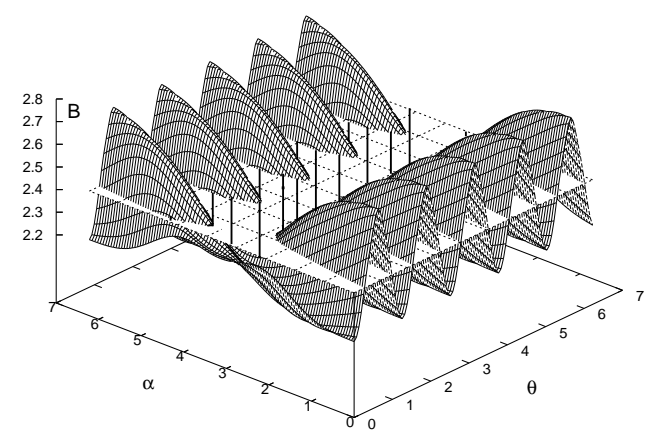

FIG. 1: Mishchenko et al. 


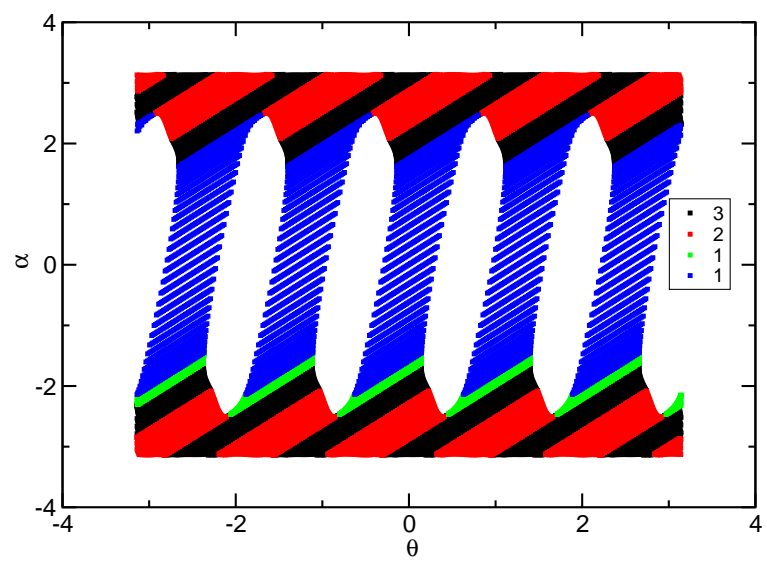

FIG. 2: Mishchenko et al. 


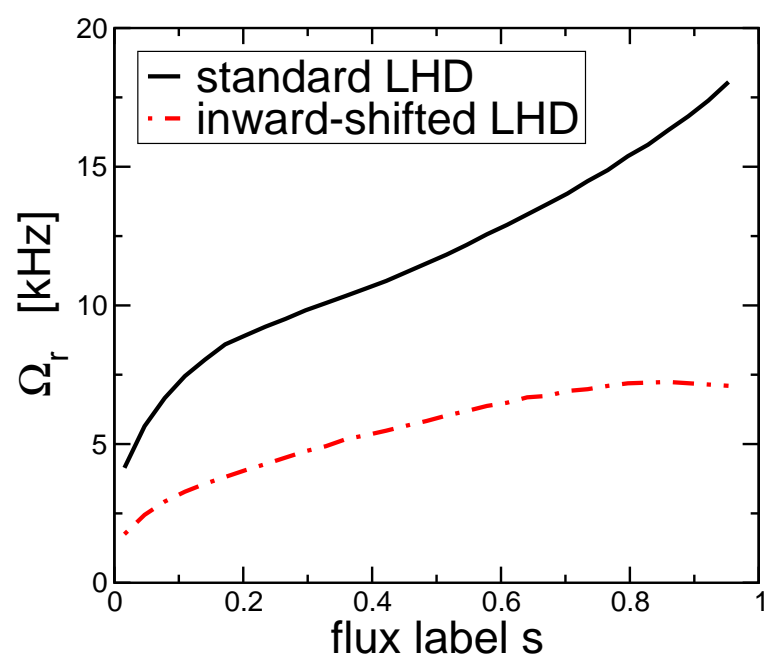

FIG. 3: Mishchenko et al. 


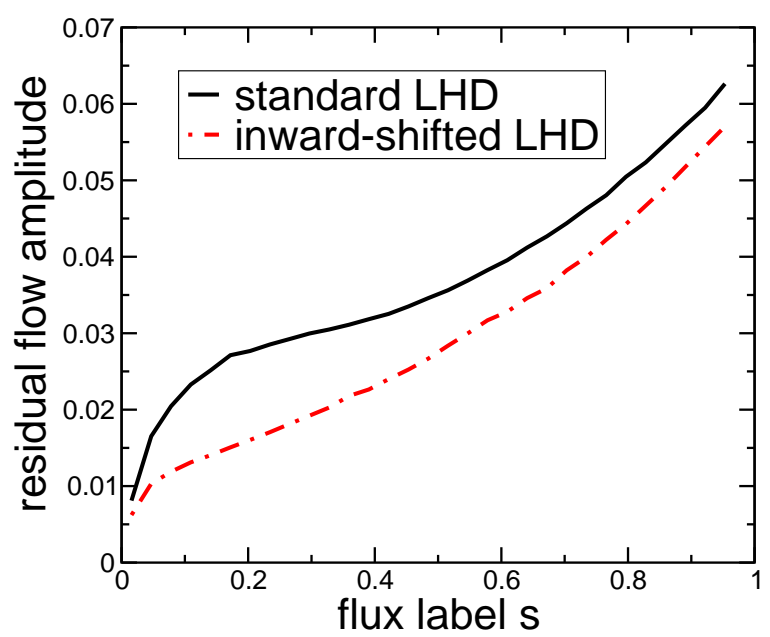

FIG. 4: Mishchenko et al. 


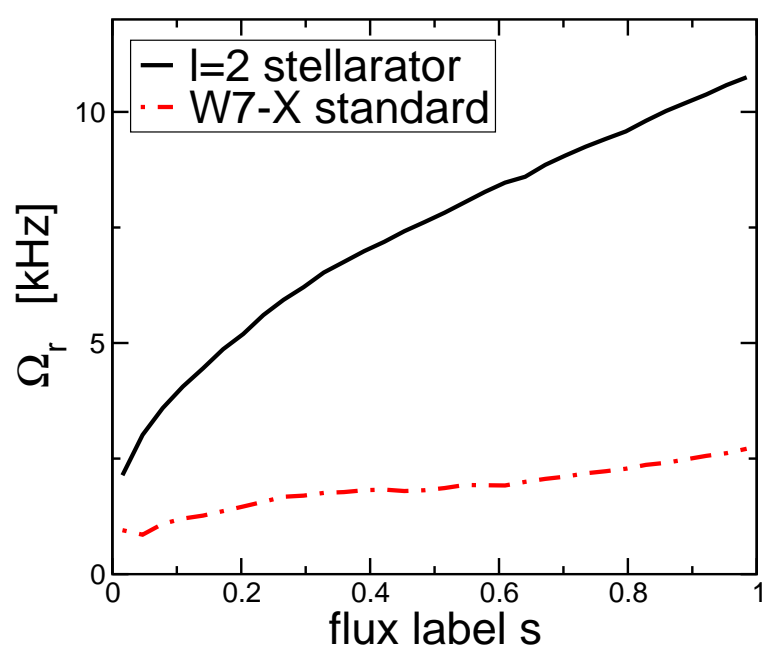

FIG. 5: Mishchenko et al. 


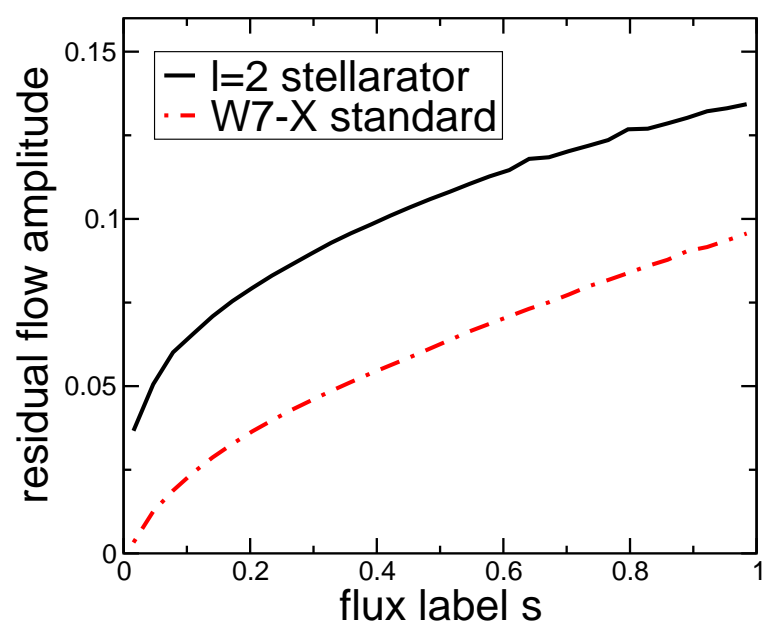

FIG. 6: Mishchenko et al. 